\title{
Morphological and Yield Traits of Pole-Type French Bean Genotypes
}

\author{
Binod Prasad Luitel ${ }^{1 @}$, Santosh Kalauni ${ }^{2}$ and Bishnu Bahadur Bhandari ${ }^{1}$
}

${ }^{1}$ Horticulture Research Station, Kimugaun, Dailekh, Nepal; @: binodsan@yahoo.com; (D):https://orcid.org/00000002-6958-4687

${ }^{2}$ Directorate of Agricultural Research, Lumbini Province, Khajura, Banke, Nepal; SK:

santoshkalauni11@gmail.com; BBB; bhandaribishnu1989@gmail.com

\author{
Received 19 September, 2020, Revised 20 February, 2021, Accepted 01 March, \\ 2021, Published 30 April, 2021 \\ Scientific Editors: UK Acharya, KC Dahal, K Upadhyay \\ Copyright $@ 2021$ NARC. Permits unrestricted use, distribution and \\ reproduction in any medium provided the original work is properly cited. \\ The authors declare that there is no conflict of interest.
}

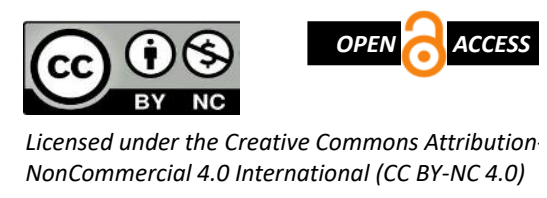

\section{ABSTRACT}

Understanding the phenotypic variation and association between the grain yield, and other agronomic traits in French bean genotypes is important for its varietal improvement program. Field experiments were conducted during 2018 and 2019 cropping season at Horticulture Research Station, Dailekh, Karnali Province of Nepal to evaluate pole-type French bean genotypes for plant morphological characters, yield and the association between the agronomic traits. Twelve (Bhatte, Chaumase, Dhankute Chhirke, WP Con Bean, White OP, Dhundi Raj, LB31, LB-37, LB-39, Madhav, Chinese Long, and Trishuli) pole-type French bean genotypes were laid out in a randomized complete block design (RCBD) with three replications. Pole-type French bean genotypes showed the significant variation for plant morphological traits and grain yield. Genotype LB-31 exhibited significantly the highest pod number (78.0/plant), green pod weight $(675.0 \mathrm{~g} / \mathrm{plant})$, green pod yield $(36.1 \mathrm{t} / \mathrm{ha})$, and dry grain yield (2.4 t/ha). Following LB-31 genotype, genotype Chaumase and LB-39 produced the highest green pod yield (31.3 t/ha and $31.2 \mathrm{t} / \mathrm{ha}$, respectively). The number of clusters per plant, pod number per plant and green pod weight per plant exhibited the significant positive correlation with green pod and dry grain yield. Hence, these traits can be selected to improve the yield potential of pole-type French bean genotypes. Pole type French bean genotypes used in the experiment were well adapted to the study area. Therefore, the high yielding genotypes viz LB-31, Chaumase and LB-39 could be recommended to use as seed source for on-farm production in Mid-Western Nepal.

Keywords: Correlation, genotypes, on-farm, phenotypic variation, yield

सारांश

घ्यू सिमीको जातहरुमा फिनोटाइपको फरक साथै दानाको उत्पादकत्व र अरु वालीविशेषताहरुको बीचको सम्बन्धलाई बुभ्नु यसको जातीय सुधार कार्यकमको लागि महत्वपूर्ण हुन्छ। वागवानी अनुसन्धान केन्द्र, दैलेखमा सन् २०१६ र २०१९ को वाली मौसममा भांगे घ्यू सिमीका जातहरुमा विरुवाको विशेषताहरु, उत्पादकत्व र वालीविशेषताहरुको बीचको सम्बन्ध मूल्याड्छन गर्नको लागि खेतमा एक परिक्षण गरिएको थियो । बाह्र वटा कांगे घ्यू सिमीका जातहरु (भट्टे, चौमासे, धनकुटे छिर्के, डब्लु पि कन विन, हाइट ओपी, ढुण्डीराज, एलवि-३१, एलवि३७, एलवि-३९, माधव, चाइनिज लङ र त्रिशुली) रयाण्डोमाइज्ड कम्लीट ब्लक डिजाइन संगै तीन वटा रेप्लिकेशनमा लगाइएको थियो । कांगे घ्यू सिमीका जातहरुले विरुवाको विशेषताहरु र दानाको उत्पादकत्वमा महत्वपूर्ण फरक देखाएको थियो। एलवि-३१ सिमीको जातले बढी हरियो कोसा (७६.०/विरुवा), हरियो कोसाको तौल (६७५.० ग्राम/विरुवा), हरिया कोसाको उत्पादकत्व (३६.१ टन/हे.) र सुख्खा दानाको उत्पादकत्व (२.४ टन/हे.) दिएको थियो । एलवि-३१ जातपछि चौमासे र एलवि-३९ जातहरुले कमश: बढ़ी हरियो कोसाको उत्पादकत्व (३१.३ टन/हे.) र (३१.२ टन/हे.) उत्पादन दियो । क्लष्टरको संख्या/विरुवा, कोसाको संख्या/विरुवा र हरियो कोसाको तौल/विरुवाले हरियो कोसा र सुख्खा दानाको उत्पादकत्वसंग महत्वपूर्ण धनात्मक सहसम्बन्ध देखायो भने यीविशेषताहरु छनोटगरि घ्यू सिमीको जातहरुको उत्पादन क्षमतामा सुधार गर्न सकिन्छ। यस परिक्षणमा प्रयोग भएका भांगे घ्यू सिमीका जातहरु अध्ययन क्षेत्रमा राम्रोसंग अनुकुलित थिए, त्यसैले बढी उत्पादनशिल जातहरु जस्तै एलवि-३१, चौमासे र एलवि-३९ किसानको खेतमा उत्पादनको लागि वीउको श्रोतको रुपमा प्रयोग गर्न मध्यपश्चिम नेपालमा सिफारिस गर्न सकिन्छ। 


\section{INTRODUCTION}

French bean (Phaseolus vulgaris L.) is a native crop of central and South America (Swaider et al 1992). This is widely cultivated in the temperate and subtropical regions and in many parts of the tropics (Pandey et al 2011). French bean is also known by different names viz snap bean, kidney bean, haricot bean, navy bean, garden bean or string bean, pole bean, bush bean, one of the most important leguminous and income generating vegetable crop of Nepal. It is grown mainly for its tender fleshy green pods, shelled green seeds and also dry beans. French bean contains considerable amount of protein being high in lysine and a good source of energy. It has anti-diabetic property and this makes a good complement staple in the diet (Singh and Singh 1992). French bean is predominantly grown in mid hills of Nepal as major vegetable crop, which is supplementing the protein source for the poor farmers of Nepal. Generally, beans are regarded as 'meat of the poor' that contributes essential protein to the undernourished people living in hills.

In Nepal, pole-type French bean is grown in an area of 4,861 ha with annual production of 50,815 ton with productivity of 10.0 t/ha (MoAD 2018). Pole type French bean is widely cultivated in almost in Nepal with a wide range of variation in altitude, rainfall, temperature, cropping system and socio-economic factors. In general, pole type French bean is commonly grown during winter and summer season. This is customary to grow with maize crop as intercrop especially in rain-fed condition at mid hills. In contrast, this is also cultivated as mono-crop in commercial level in peri-urban areas. Most of pole type French bean genotypes grown in Nepal are introduced either from India or China but farmers in hills and mountain have been growing a number of landraces with varying morphologies (Neupane and Vaidya 2002).

Pole type French bean genotypes grown in hills so far are low yielding type and assessment of variability in the introduced and locally collected landraces can possibly enable to identify the elite genotypes. Yield is complex quantitative trait which is controlled by many yield related traits (Alemu et al 2017). Pod length, diameter, number of seed and pods in plant are the most crucial traits for plant yield (Stoilova et al 2005). The development of improved varieties in French bean is one of the most important task to meet increasing demand of stakeholders. Genotype is the most potent factor in any crop production program and is the basic material to which all other technologies are applied (Goutam et al 2001). Genotypes also greatly vary on their performance under different agro-climatic condition. Understanding the phenotypic variability, association among the phenotypic traits and other qualitative traits is necessary for the variety improvement program in French bean. This study was conducted to evaluate pole type French bean genotypes for morphological, yield traits, association among the quantitative traits and other qualitative traits as well.

\section{MATERIALS AND METHODS}

\section{Experimental Site}

Field experiments were conducted during the winter season of 2018 and 2019 at Horticulture Research Station (HRS), Dailekh in mid-western Nepal. The geographical coordinates of the site are $28^{\circ} 13^{\prime} 6.18^{\prime \prime} \mathrm{N}$ latitude and $83^{\circ} 58^{\prime} 27.72 " E$ longitude with an altitude of 1290 meters above mean sea level. The soil of experimental field was characterized by loamy type. In the year 2018, average $9.1 \mathrm{~mm}$ rainfall was received in September. In contrast, the highest $(400.8 \mathrm{~mm})$ rainfall was received in September, 2019 (Figure1). Amount of rainfall received was higher in 2019 as compared to 2018 during September-December. The maximum temperature in the years 2018 and 2019 was decreased from September to December and similar trend was observed in minimum temperature in the year 2018. But compared to minimum temperature of each month in the year 2018, it was considerably increased at each month in the year 2019 (Figure1). 


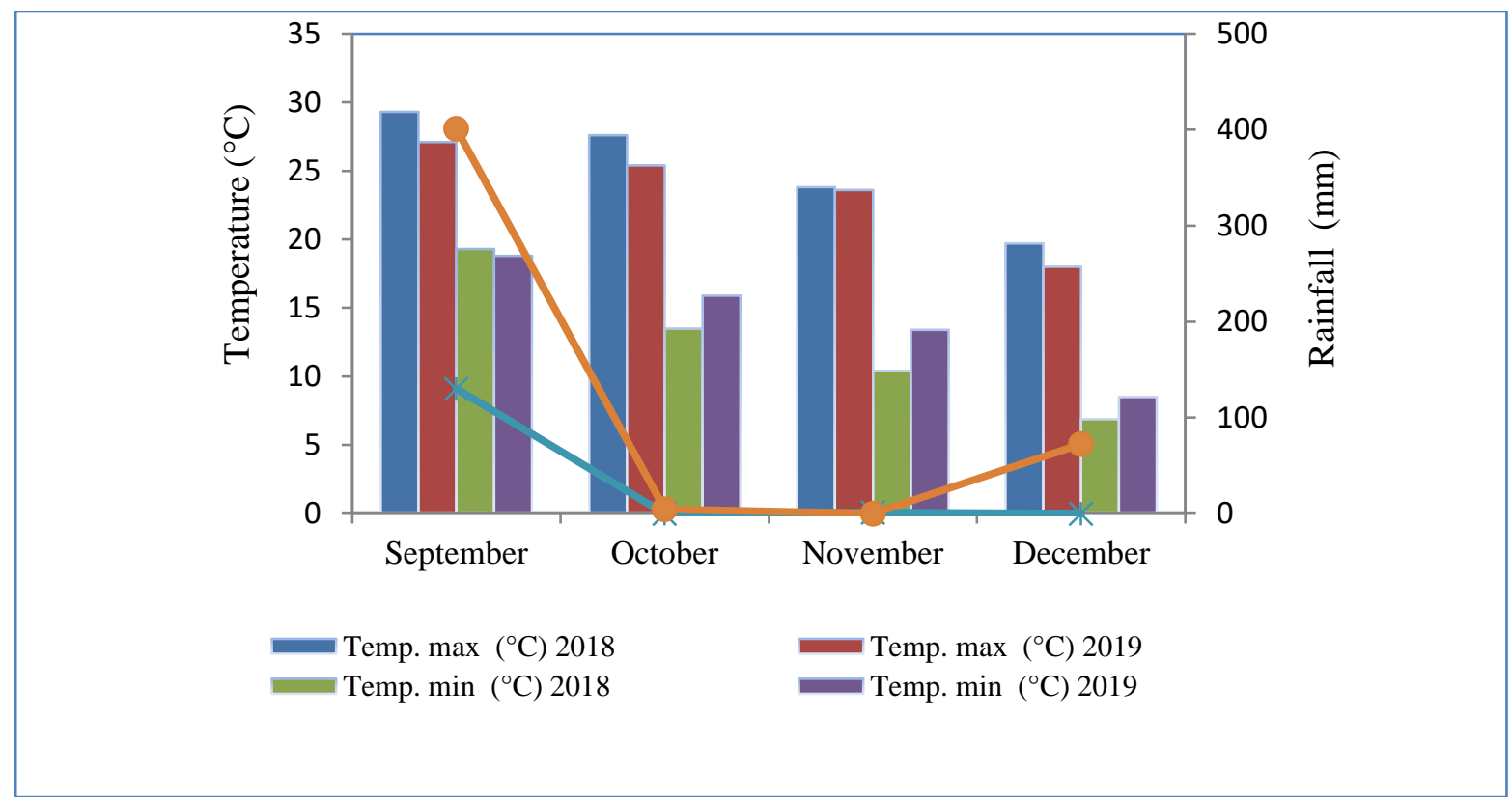

Figure1. Monthly weather during winter season of 2018 and 2019 experimentation period at Dailekh, Nepal

\section{Treatments and Experimental Design}

Treatments consisted of twelve genotypes (Bhatte, Chaumase, Dhankute Chhirke, WP Con Bean, White OP, Dhundi Raj, LB-31, LB-37, LB-39, Madhav, Chinese Long, and Trishuli) were used in both years. Trishuli, a released and popular pole bean variety in mid-hills, was used as check variety. The treatments were arranged in a randomized complete block design (RCBD) with three replications. Experimental field was ploughed, pulverized and leveled in order to get smooth seedbed and inter-and intra-row spacing was maintained at $70 \mathrm{~cm}$ x $25 \mathrm{~cm}$, respectively. In both the years 2018 and 2019, the plot size was maintained $6 \mathrm{~m}^{2}$ ( $2 \mathrm{~m}$ wide and $3 \mathrm{~m}$ long). Seeds were hand planted by placing two seeds/hill. In 2018, seeding was done in September 2 and first harvested in November 21. Whereas in 2019, seeding was done in August 30 and the trial was first harvested in Nov. 28. Thirty-two plants per plot were maintained in four rows. The recommended $\mathrm{N}: \mathrm{P}_{2} \mathrm{O}_{5}: \mathrm{K}_{2} \mathrm{O}$ fertilizers were applied at planting time at the rate of $80: 120: 60 \mathrm{~kg} / \mathrm{ha}$, respectively and urea was used as $\mathrm{N}$ source. Manure was applied as compost (20 t/ha) and the half portion of nitrogen was applied as top-dressing after 45 days of seed sowing. All crop management practices such as cultivation, weeding etc carried out as desired during crop growing period.

\section{Data Collection, Measurements and Statistical Analysis}

Data on plant height $(\mathrm{cm})$, cluster per plant (no.), and pod per plant (no.) were recorded on randomly selected five sample plants and averaged it. Plant height $(\mathrm{cm})$ was measured using meter-scale at physiological maturity stage from cotyledon scar to tip of the plant. Individual green pod weight was taken on 10 pods using digital weighing balance and averaged it. Green pod weight per plant (g) was recorded on randomly selected five sample plants. Green pod weight per plot $(\mathrm{kg})$, and green pod yield ( $\mathrm{t} / \mathrm{ha})$ were obtained by adding the total weight of three harvests from two rows $\left(3 \mathrm{~m}^{2}\right.$ plot $)$. Pod length $(\mathrm{cm})$ and diameter ( $\mathrm{mm}$ ) was measured using meter-scale and Vernier caliper, respectively, in the largest fully expanded immature pods from five random normal plants. Similarly, dry pod per plant (no.), and dry pod weight per plant $(\mathrm{g})$ were measured on randomly selected five plants and averaged it. The seed per pod (no.) was recorded on five pods from five plants and averaged it. Dry pod weight per plot (g) was calculated by adding the total weight of three harvests from two rows. After sun drying of grain and maintaining moisture content upto $12-14 \%$, 100 seeds were counted and weighed. Then, dry grain weight per plot (g) and dry grain yield ( $\mathrm{t} / \mathrm{ha}$ ) was calculated. The qualitative traits including flower color, pod beak orientation, pod color, pod quality, seed color and seed shape were taken according to the procedures given in the 
International Board for Plant Genetic Resources (IBPGR) descriptors for Phaseolus vulgaris (IBPGR 1982). Flower color was observed in freshly open flower. Pod beak orientation (upward, straight or downward) was recorded at green pod maturity stage by visual observation. Pod color was recorded on the fully expanded immature pods as green, light green, green and red stripes, green and light purple stripes, and green and purple stripes. Pod quality was assessed by visual observation. Seed was taken from middle of the dry pod and seed shape was recorded as circular to elliptic, elliptic, and kidney. The quantitative data were subjected to analysis of variance (ANOVA)using GenStat Release 10.3 DE Software (VSN International, Hemel Hempstead,UK).The genotype, year and their interaction effects were analyzed by combined ANOVA and pooled data over the years are presented. Correlation among the quantitative variables was analyzed using IBM SPSS Statistics (Version 20). The qualitative data were expressed as tabulated and pictorial form wherever necessary.

\section{RESULTS}

\section{Plant Characters}

The pooled data over the years showed that the plant height was highly significant $(p \leq 0.01)$ among the genotypes (Table1). The average plant height was higher $(257.6 \mathrm{~cm})$ in 2019 than the year $2018(209.7$ $\mathrm{cm})$. The highest $(279.2 \mathrm{~cm})$ plant height was measured in Dhundi Raj followed by LB-37 $(264.0 \mathrm{~cm})$, LB$39(255.0 \mathrm{~cm})$, Madhav $(254.7 \mathrm{~cm})$ and the lowest $(181.8 \mathrm{~cm})$ was measured in Dhankute Chhirke. The number of clusters per plant had non-significant effect among the genotypes. But year had highly significant effect on number of clusters produced per plant. The number of clusters produced per plant was higher (27.12) in the year 2019 than the year 2018 (15.82). Genotypes had highly significant $(p \leq 0.01)$ effect on pod number per plant. The highest (78.0) number of pods per plant was counted in LB-31 followed by Chaumase (70.0), and LB-37 (66.0) whereas the lowest (35.0) pod number per plant was measured in LB39.

Table 1. Plant morphological characters of pole-type French bean genotypes during the years 2018 and 2019

\begin{tabular}{|c|c|c|c|c|c|c|c|c|c|}
\hline \multirow[t]{2}{*}{ Genotype } & \multicolumn{3}{|c|}{ Plant height $(\mathbf{c m})$} & \multicolumn{3}{|c|}{ Cluster/plant (no.) } & \multicolumn{3}{|c|}{ Pod/plant (no.) } \\
\hline & 2018 & 2019 & Mean & 2018 & 2019 & Mean & 2018 & 2019 & Mean \\
\hline Bhatte & 174.7 & 248.3 & 211.5 & 12.0 & 31.0 & 22.0 & 49.0 & 73.0 & 61.0 \\
\hline Chaumase & 187.7 & 222.7 & 205.2 & 19.0 & 22.0 & 21.0 & 94.0 & 46.0 & 70.0 \\
\hline DhankuteChhirke & 137.7 & 206.0 & 181.8 & 12.0 & 28.0 & 20.0 & 55.0 & 63.0 & 59.0 \\
\hline WP Con Bean & 177.0 & 228.3 & 202.7 & 14.0 & 35.0 & 24.0 & 58.0 & 53.0 & 56.0 \\
\hline White OP & 213.7 & 279.0 & 246.3 & 15.0 & 26.0 & 20.0 & 68.0 & 54.0 & 61.0 \\
\hline Dhundi Raj & 282.7 & 275.7 & 279.2 & 16.0 & 30.0 & 23.0 & 60.0 & 40.0 & 50.0 \\
\hline LB-31 & 206.5 & 282.0 & 243.3 & 20.0 & 32.0 & 26.0 & 88.0 & 67.0 & 78.0 \\
\hline LB-37 & 268.7 & 259.3 & 264.0 & 20.0 & 28.0 & 24.0 & 78.0 & 53.0 & 66.0 \\
\hline LB-39 & 224.0 & 286.0 & 255.0 & 13.0 & 21.0 & 17.0 & 31.0 & 39.0 & 35.0 \\
\hline Madhav & 246.0 & 263.3 & 254.7 & 13.0 & 24.0 & 19.0 & 69.0 & 42.0 & 56.0 \\
\hline Chinese Long & 191.7 & 265.0 & 228.3 & 16.0 & 25.0 & 20.0 & 56.0 & 47.0 & 52.0 \\
\hline Trishuli (Ch) & 205.7 & 276.0 & 240.8 & 21.0 & 25.0 & 23.0 & 61.0 & 39.0 & 50.0 \\
\hline Mean & 209.7 & 257.6 & 233.6 & 15.82 & 27.12 & 21.47 & 63.9 & 51.5 & 57.7 \\
\hline Genotype (G) & & & $* *$ & & & NS & & & $* *$ \\
\hline Year (Y) & & & $* *$ & & & ** & & & $* *$ \\
\hline $\mathrm{G} \times \mathrm{Y}$ & & & NS & & & NS & & & $*$ \\
\hline $\operatorname{LSD}(0.05)$ & & & 42.15 & & & 7.67 & & & 18.4 \\
\hline $\mathrm{CV}(\%)$ & & & 15.5 & & & 30.8 & & & 27.4 \\
\hline
\end{tabular}

NS $=$ Non-significant; $*$ and $* *$ Significant at 0.05 and 0.01 levels, respectively.

\section{Green Pod Weight and Yield}

Data of green pod weight per pod, plant, plot and yield are mentioned in Table 2. Genotypes had highly significant $(p \leq 0.01)$ effect on green pod weight per pod. LB-39 produced the highest green pod weight per 
pod in both years 2018 and 2019 and the values were $20.6 \mathrm{~g}$ and $20.8 \mathrm{~g}$, respectively. The green pod weight was recorded the highest (20.8 g) in LB-39 followed by Trishuli (16.6 g) and the lowest (7.4 g) was recorded in Dhankute Chhirke. Greed pod weight per plant, green pod weight per plot and green pod yield was significantly $(p \leq 0.05)$ different among the genotypes. Genotype LB-31 yielded the highest green pod weight per plant, green pod weight per plot and green pod yield in both years. The highest $(675.0 \mathrm{~g})$ green pod weight per plant was measured in LB-31 followed by Chaumase (587.0 g) whereas the lowest green pod weight $(330.0 \mathrm{~g})$ was measured in Dhankute Chhirke. The highest $(10.8 \mathrm{~kg})$ green pod weight per plot was weighted in LB-31 followed by Chaumase $(9.4 \mathrm{~kg})$ and LB-39 $(9.4 \mathrm{~kg})$ whereas the lowest $(5.3 \mathrm{~kg})$ was in Dhankute Chhirke. LB-31 produced significantly the highest (36.1 t/ha) green pod yield followed by Chaumase (31.3 t/ha) and LB-39 (31.2 t/ha) and the lowest (17.6 t/ha) yield was recorded in Dhankute Chhirke.

Table 2. Green pod weight and yield of pole-type French bean genotypes during the years 2018 and 2019

\begin{tabular}{|c|c|c|c|c|c|c|c|c|c|c|c|c|}
\hline \multirow[t]{2}{*}{ Genotype } & \multicolumn{3}{|c|}{ Green pod wt./pod (g) } & \multicolumn{3}{|c|}{$\begin{array}{l}\text { Green pod wt./plant } \\
\text { (g) }\end{array}$} & \multicolumn{3}{|c|}{ Green pod wt./plot (kg) } & \multicolumn{3}{|c|}{ Greed pod yield (t/ha) } \\
\hline & 2018 & 2019 & Mean & 2018 & 2019 & Mean & 2018 & 2019 & Mean & 2018 & 2019 & Mean \\
\hline Bhatte & 9.3 & 11.1 & 10.2 & 303.0 & 625.0 & 464.0 & 4.8 & 10.0 & 7.4 & 16.2 & 33.4 & 24.8 \\
\hline Chaumase & 9.8 & 10.1 & 9.9 & 568.0 & 605.0 & 587.0 & 9.1 & 9.7 & 9.4 & 30.3 & 32.3 & 31.3 \\
\hline DhankuteChhirke & 7.8 & 6.8 & 7.4 & 261.0 & 400.0 & 330.0 & 4.2 & 6.4 & 5.3 & 13.9 & 21.3 & 17.6 \\
\hline WP Con Bean & 8.5 & 8.9 & 8.7 & 428.0 & 384.0 & 406.0 & 6.8 & 6.1 & 6.5 & 22.8 & 20.4 & 21.6 \\
\hline White OP & 9.8 & 8.5 & 9.2 & 465.0 & 388.0 & 426.0 & 7.4 & 6.2 & 6.8 & 24.8 & 20.6 & 22.7 \\
\hline Dhundi Raj & 10.8 & 15.0 & 12.9 & 351.0 & 476.0 & 415.0 & 5.7 & 7.6 & 6.6 & 18.9 & 25.3 & 22.2 \\
\hline LB-31 & 13.2 & 11.7 & 12.5 & 634.0 & 716.0 & 675.0 & 10.2 & 11.5 & 10.8 & 33.8 & 38.2 & 36.1 \\
\hline LB-37 & 10.1 & 12.2 & 11.1 & 500.0 & 468.0 & 484.0 & 8.0 & 7.5 & 7.8 & 26.7 & 24.9 & 25.8 \\
\hline LB-39 & 20.6 & 20.8 & 20.8 & 575.0 & 595.0 & 585.0 & 9.2 & 9.5 & 9.4 & 30.6 & 31.7 & 31.2 \\
\hline Madhav & 11.8 & 12.7 & 12.3 & 526.0 & 477.0 & 502.0 & 8.4 & 7.6 & 8.0 & 28.1 & 25.4 & 26.8 \\
\hline Chinese Long & 12.4 & 12.6 & 12.5 & 345.0 & 479.0 & 412.0 & 5.5 & 7.6 & 6.6 & 18.4 & 25.5 & 21.9 \\
\hline Trishuli (Ch) & 15.8 & 17.3 & 16.6 & 453.0 & 504.0 & 479.0 & 7.3 & 8.1 & 7.6 & 24.2 & 26.9 & 25.5 \\
\hline Mean & 11.67 & 12.33 & 12.0 & 450.0 & 510.0 & 480.0 & 7.22 & 8.16 & 7.69 & 24.06 & 27.2 & 25.6 \\
\hline Genotype (G) & & & ** & & & $*$ & & & $*$ & & & $*$ \\
\hline Year (Y) & & & NS & & & NS & & & NS & & & NS \\
\hline $\mathrm{G} \times \mathrm{Y}$ & & & NS & & & NS & & & NS & & & NS \\
\hline LSD (0.05) & & & 1.838 & & & 168.2 & & & 2.69 & & & 8.9 \\
\hline $\mathrm{CV}(\%)$ & & & 13.2 & & & 30.1 & & & 30.1 & & & 30.1 \\
\hline
\end{tabular}

NS = Non-significant; * and ** Significant at 0.05 and 0.01 levels, respectively.

\section{Pod Characters}

Genotype showed highly significant $(p \leq 0.01)$ differences on pod length, pod diameter but it had significantly $(p \leq 0.05)$ different on dry pod number per plant (Table 3$)$. The pooled data over the years showed that the longest $(22.3 \mathrm{~cm})$ pod length was measured in LB-39 and the lowest $(10.8 \mathrm{~cm})$ pod length was measured in Dhankute Chhirke. In contrast, the highest pod diameter was measured in Bhatte (11.9 $\mathrm{mm})$ followed by LB-39 $(11.6 \mathrm{~mm})$ and Dhankute Chhirke $(11.5 \mathrm{~mm})$ and the lowest $(9.1 \mathrm{~mm})$ diameter was measured in WP Con Bean. The combined analysis showed that LB-31 produced the highest (32.0) number of dry pod per plant followed by Madhav (31.0) and the lowest (15.0) dry pod number was counted in LB-39. 
Table 3. Pod morphological characters of pole-type French bean genotypes during the years 2018 and 2019 at HRS, Dailekh

\begin{tabular}{|c|c|c|c|c|c|c|c|c|c|}
\hline \multirow[t]{2}{*}{ Genotype } & \multicolumn{3}{|c|}{ Pod length $(\mathrm{cm})$} & \multicolumn{2}{|c|}{ Pod dia. (mm) } & \multicolumn{4}{|c|}{ Dry pod/plant (no.) } \\
\hline & 2018 & 2019 & Mean & 2018 & 2019 & Mean & 2018 & 2019 & Mean \\
\hline Bhatte & 12.0 & 14.8 & 13.4 & 10.1 & 13.8 & 11.9 & 31.0 & 27.0 & 29.0 \\
\hline Chaumase & 15.9 & 16.0 & 15.9 & 9.1 & 9.5 & 9.3 & 38.0 & 19.0 & 28.0 \\
\hline Dhankute Chhirke & 10.2 & 11.3 & 10.8 & 10.5 & 12.4 & 11.5 & 24.0 & 17.0 & 21.0 \\
\hline WP Con Bean & 13.6 & 15.8 & 14.7 & 9.0 & 9.2 & 9.1 & 27.0 & 31.0 & 29.0 \\
\hline White OP & 13.9 & 15.8 & 14.9 & 9.2 & 9.3 & 9.2 & 36.0 & 18.0 & 27.0 \\
\hline Dhundi Raj & 16.8 & 19.0 & 17.9 & 9.8 & 11.8 & 10.8 & 26.0 & 21.0 & 23.0 \\
\hline LB-31 & 16.7 & 18.5 & 17.6 & 9.9 & 10.2 & 10.1 & 38.0 & 25.0 & 32.0 \\
\hline LB-39 & 21.4 & 23.1 & 22.3 & 10.8 & 12.4 & 11.6 & 15.0 & 14.0 & 15.0 \\
\hline LB-37 & 16.3 & 19.5 & 17.8 & 10.1 & 12.9 & 10.5 & 27.0 & 20.0 & 24.0 \\
\hline Madhav & 15.9 & 18.2 & 17.1 & 9.8 & 11.24 & 10.6 & 39.0 & 24.0 & 31.0 \\
\hline Chinese Long & 16.6 & 17.8 & 17.2 & 9.7 & 10.9 & 10.4 & 26.0 & 21.0 & 23.0 \\
\hline Trishuli (Ch) & 18.1 & 20.8 & 19.4 & 10.3 & 11.6 & 10.9 & 29.0 & 13.0 & 21.0 \\
\hline Mean & 15.6 & 17.58 & 16.6 & 9.88 & 11.12 & 10.5 & 29.6 & 20.8 & 25.2 \\
\hline Genotype (G) & & & $* *$ & & & $* *$ & & & * \\
\hline Year $(Y)$ & & & $* *$ & & & $* *$ & & & $* *$ \\
\hline $\mathrm{G} \times \mathrm{Y}$ & & & NS & & & $* *$ & & & NS \\
\hline $\operatorname{LSD}(0.05)$ & & & 1.329 & & & 0.656 & & & 10.03 \\
\hline $\mathrm{CV}(\%)$ & & & 6.9 & & & 5.4 & & & 34.2 \\
\hline
\end{tabular}

NS = Non-significant; $*$ and $* *$ Significant at 0.05 and 0.01 levels, respectively.

Dry Pod and Seed Characters

Data on dry pod weight per plant, seed number per pod, and dry pod weight per plot are presented in Table 4. Dry pod weight per plant was non-significant among the genotypes whereas seed number per pod was highly significant $(p \leq 0.01)$. The number of seeds per pod produced the highest (8.0) in Trishuli variety in 2018 but it produced the highest (9.0) in LB-37 in 2019.

Table 4. Pod and seed morphological characters of pole-type French bean genotypes during the years 2018 and 2019 at HRS, Dailekh

\begin{tabular}{|c|c|c|c|c|c|c|c|c|c|}
\hline \multirow[t]{2}{*}{ Genotype } & \multicolumn{3}{|c|}{ Dry pod wt./plant (g) } & \multicolumn{3}{|c|}{ Seed/pod (no.) } & \multicolumn{3}{|c|}{ Dry pod wt./plot (g) } \\
\hline & 2018 & 2019 & Mean & 2018 & 2019 & Mean & 2018 & 2019 & Mean \\
\hline Bhatte & 65.4 & 71.8 & 68.6 & 3.0 & 7.0 & 5.0 & 591.0 & 1149.0 & 870.0 \\
\hline Chaumase & 80.1 & 59.0 & 69.6 & 7.0 & 8.0 & 8.0 & 795.0 & 945.0 & 870.0 \\
\hline DhankuteChhirke & 81.7 & 56.4 & 69.0 & 5.0 & 5.0 & 5.0 & 825.0 & 902.0 & 864.0 \\
\hline WP Con Bean & 36.7 & 59.7 & 48.2 & 6.0 & 8.0 & 7.0 & 342.0 & 956.0 & 649.0 \\
\hline White OP & 42.9 & 50.0 & 46.4 & 6.0 & 8.0 & 7.0 & 401.0 & 800.0 & 600.0 \\
\hline Dhundi Raj & 62.9 & 58.3 & 60.6 & 7.0 & 7.0 & 7.0 & 820.0 & 933.0 & 877.0 \\
\hline LB-31 & 64.3 & 79.2 & 71.8 & 5.0 & 8.0 & 7.0 & 875.0 & 1268.0 & 1071.0 \\
\hline LB-37 & 65.9 & 79.0 & 72.5 & 7.0 & 9.0 & 8.0 & 945.0 & 1264.0 & 1105.0 \\
\hline LB-39 & 46.0 & 35.8 & 40.9 & 7.0 & 8.0 & 8.0 & 416.0 & 572.0 & 494.0 \\
\hline Madhav & 70.6 & 60.3 & 65.4 & 6.0 & 8.0 & 8.0 & 758.0 & 965.0 & 861.0 \\
\hline Chinese Long & 57.0 & 61.3 & 59.1 & 7.0 & 8.0 & 8.0 & 371.0 & 981.0 & 676.0 \\
\hline Trishuli (Ch) & 82.6 & 60.3 & 71.5 & 8.0 & 8.0 & 8.0 & 1070.0 & 965.0 & 1018.0 \\
\hline Mean & 63.0 & 60.9 & 62.0 & 6.19 & 7.83 & 7.0 & 684.0 & 975.0 & 830.0 \\
\hline Genotype (G) & & & NS & & & ** & & & * \\
\hline Year (Y) & & & NS & & & $* *$ & & & $* *$ \\
\hline $\mathrm{G} \times \mathrm{Y}$ & & & NS & & & $* *$ & & & NS \\
\hline LSD (0.05) & & & 27.73 & & & 0.783 & & & 339.4 \\
\hline CV (\%) & & & 38.5 & & & 9.6 & & & 35.2 \\
\hline
\end{tabular}


The pooled mean showed the highest (8.0) seed number per pod in Chaumase, LB-37, LB-39, Madhav, Chinese Long and Trishuli and the lowest (5.0) seed per pod produced was in Bhatte and Dhankute Chhirke. Dry pod weight per plot was significantly $(p \leq 0.05)$ different among the genotypes. The dry pod weight per plot was produced the highest $(1070.0 \mathrm{~g})$ in Trishuli in 2018 whereas LB-31 produced the highest (1268.0 g) dry pod weight per plot in 2019. However, the pooled mean over the years showed the highest (1105.0 g) dry pod weight per plot in LB-37 but it was statistically similar at LB-31 (1071.0 g), Trishuli (1018.0 g), LB-31 (877.0 g), Bhatte (870.0 g) and Chaumase (870.0 g).

\section{Dry Grain Weight and Yield}

Dry grain weight per plot, hundred grain weight and dry grain yield were highly significant $(p \leq 0.01)$ among French bean genotypes (Table 5). The pooled mean over the years showed the highest (720.0 g) dry grain weight per plot which was statistically at par with LB-31 (716.0 g), Chaumase (646.0 g) and LB-37 (633.0 $\mathrm{g})$ and the lowest $(330.0 \mathrm{~g})$ dry grain weight per plot was produced in WP Con Bean. With respect to hundred grain weight, Dhankute Chhirke yielded the highest $(53.0 \mathrm{~g})$ followed by LB-37 $(43.5 \mathrm{~g})$ but the minimum hundred grain weight was produced in White OP $(18.6 \mathrm{~g})$. With regard to dry grain yield, genotype LB-31 and Trishuli produced the highest (2.4 t/ha) but it was statistically similar with Chaumase (2.2 t/ha) and LB-37 (2.1 t/ha) and the lowest (1.1 t/ha) grain yield was recorded in White OP.

Table 5. Dry grain weight and yield characters of pole-type French bean genotypes during the years 2018 and 2019 at HRS, Dailekh

\begin{tabular}{|c|c|c|c|c|c|c|c|c|c|}
\hline \multirow[t]{2}{*}{ Genotype } & \multicolumn{2}{|c|}{$\begin{array}{c}\text { Dry grain wt./plot } \\
\text { (g) }\end{array}$} & \multicolumn{4}{|c|}{100 grain wt. (g) } & \multicolumn{3}{|c|}{ Dry grain yield (t/ha) } \\
\hline & 2018 & 2019 & Mean & 2018 & 2019 & Mean & 2018 & 2019 & Mean \\
\hline Bhatte & 416.0 & 690.0 & 553.0 & 32.5 & 32.7 & 32.6 & 1.4 & 2.3 & 1.8 \\
\hline Chaumase & 810.0 & 481.0 & 646.0 & 25.3 & 23.0 & 24.1 & 1.6 & 2.7 & 2.2 \\
\hline Dhankute Chhirke & 555.0 & 504.0 & 530.0 & 53.8 & 52.3 & 53.0 & 1.9 & 1.7 & 1.8 \\
\hline WP Con Bean & 290.0 & 445.0 & 368.0 & 19.2 & 18.0 & 18.6 & 0.9 & 1.5 & 1.2 \\
\hline White OP & 356.0 & 303.0 & 330.0 & 19.1 & 20.0 & 19.5 & 1.2 & 1.0 & 1.1 \\
\hline Dhundi Raj & 466.0 & 384.0 & 415.0 & 37.0 & 32.7 & 33.4 & 1.5 & 1.2 & 1.4 \\
\hline LB-31 & 777.0 & 656.0 & 716.0 & 27.3 & 27.0 & 27.1 & 2.5 & 2.2 & 2.4 \\
\hline LB-37 & 683.0 & 583.0 & 633.0 & 45.3 & 41.7 & 43.5 & 2.3 & 1.9 & 2.1 \\
\hline LB-39 & 628.0 & 443.0 & 535.0 & 30.3 & 30.3 & 30.3 & 2.0 & 1.5 & 1.8 \\
\hline Madhav & 575.0 & 523.0 & 549.0 & 24.4 & 27.7 & 26.0 & 1.9 & 1.7 & 1.8 \\
\hline Chinese Long & 302.0 & 411.0 & 357.0 & 24.8 & 29.7 & 27.2 & 1.0 & 1.4 & 1.2 \\
\hline Trishuli (Ch) & 940.0 & 501.0 & 720.0 & 37.8 & 36.7 & 37.3 & 3.1 & 1.7 & 2.4 \\
\hline Mean & 567.0 & 492.0 & 529.0 & 31.13 & 30.97 & 31.05 & 1.8 & 1.6 & 1.8 \\
\hline Genotype (G) & & & $* *$ & & & $* *$ & & & $* *$ \\
\hline Year (Y) & & & NS & & & NS & & & NS \\
\hline $\mathrm{G} \times \mathrm{Y}$ & & & NS & & & NS & & & NS \\
\hline LSD (0.05) & & & 208.7 & & & 2.75 & & & 0.695 \\
\hline CV $(\%)$ & & & 33.9 & & & 7.6 & & & 33.9 \\
\hline
\end{tabular}

$N S=$ Non-significant; and $* *$ Significant at 0.01 level, respectively.

\section{Correlation among the Quantitative Characters}

The direction and magnitude of associations among the quantitative characters are given in Table 6. Plant height showed the positive and highly significant $(p \leq 0.01)$ correlation with pod length $(\mathrm{r}=0.55)$ and dry pod weight per plant $(\mathrm{r}=0.54)$. The number of clusters per plant was highly significantly $(p \leq 0.01)$ correlated with green pod weight per plant $(\mathrm{r}=0.45)$, green pod weight per plot $(\mathrm{r}=0.55)$, green pod yield $(r=0.48)$, dry pod weight per plant, $(r=0.44)$, dry pod weight per plot $(r=0.46)$, dry grain weight per plot $(\mathrm{r}=0.68)$, and dry grain yield $(\mathrm{r}=0.66)$ in positive direction. Likewise, pod number per plant exhibited the positive and highly significant $(p \leq 0.01)$ association with green pod weight per plant $(\mathrm{r}=0.48)$, green pod weight per plot $(r=0.49)$, green pod yield $(r=0.49)$, dry pod number per plant $(r=0.59)$, seed per plant $(r$ 
Table7. Evaluation of qualitative characters of pole-type French bean genotypes at HRS, Dailekh, 2018

\begin{tabular}{|c|c|c|c|c|}
\hline Genotype & $\begin{array}{l}\text { Pod beak } \\
\text { orientation }\end{array}$ & Pod color & Pod quality & Seed shape \\
\hline Bhatte & Upward & Green & Non-slender, flat, slightly smooth & Circular to elliptic \\
\hline Chaumase & Downward & Green & Stringly, slender, slightly curved, & Elliptic \\
\hline DhankuteChhirke & Upward & $\begin{array}{l}\text { Green and red } \\
\text { stripes }\end{array}$ & $\begin{array}{l}\text { Non-slender, flat, rough and small } \\
\text { seeded }\end{array}$ & Circular to elliptic \\
\hline WP Con Bean & Upward & Light green & $\begin{array}{l}\text { Smooth surface, slender, cylindrical, } \\
\text { small sized seed }\end{array}$ & Kidney \\
\hline White OP & Upward & Light green & $\begin{array}{l}\text { Slender, cylinder, smooth, big } \\
\text { seeded, straight }\end{array}$ & Kidney \\
\hline Dhundi Raj & Upward & $\begin{array}{l}\text { Green and Light } \\
\text { purple stripes }\end{array}$ & Slender, flat, rough, small seed & Kidney \\
\hline LB-31 & Downward & $\begin{array}{l}\text { Green and purple } \\
\text { stripes }\end{array}$ & $\begin{array}{l}\text { Stringy, non-slender, slightly } \\
\text { curved, flat, medium seeded }\end{array}$ & Elliptic \\
\hline LB-37 & Upward & Green & $\begin{array}{l}\text { Stringy, non-slender, slightly } \\
\text { curved, flat, slightly rough }\end{array}$ & Kidney \\
\hline LB-39 & Upward & Green & $\begin{array}{l}\text { Double stringy, straight, flat, and } \\
\text { large seeded }\end{array}$ & Kidney \\
\hline Madhav & Upward & Green & $\begin{array}{l}\text { Smooth, slender, straight, flat, small } \\
\text { seeded }\end{array}$ & Elliptic \\
\hline Chinese Long & Downward & Green & $\begin{array}{l}\text { Slender, cylindrical, smooth and } \\
\text { small seeded }\end{array}$ & Kidney \\
\hline Trishuli (Ch) & Straight & Green & $\begin{array}{l}\text { Rough surface, curved, slight } \\
\text { cylindrical }\end{array}$ & Elliptic \\
\hline
\end{tabular}

The genotypes Bhatte, WP Con bean, White OP, LB-37, LB-39 and Trishuli were characterized as white petal color (Figure 2) whereas other genotypes contained purple to violet petal color.
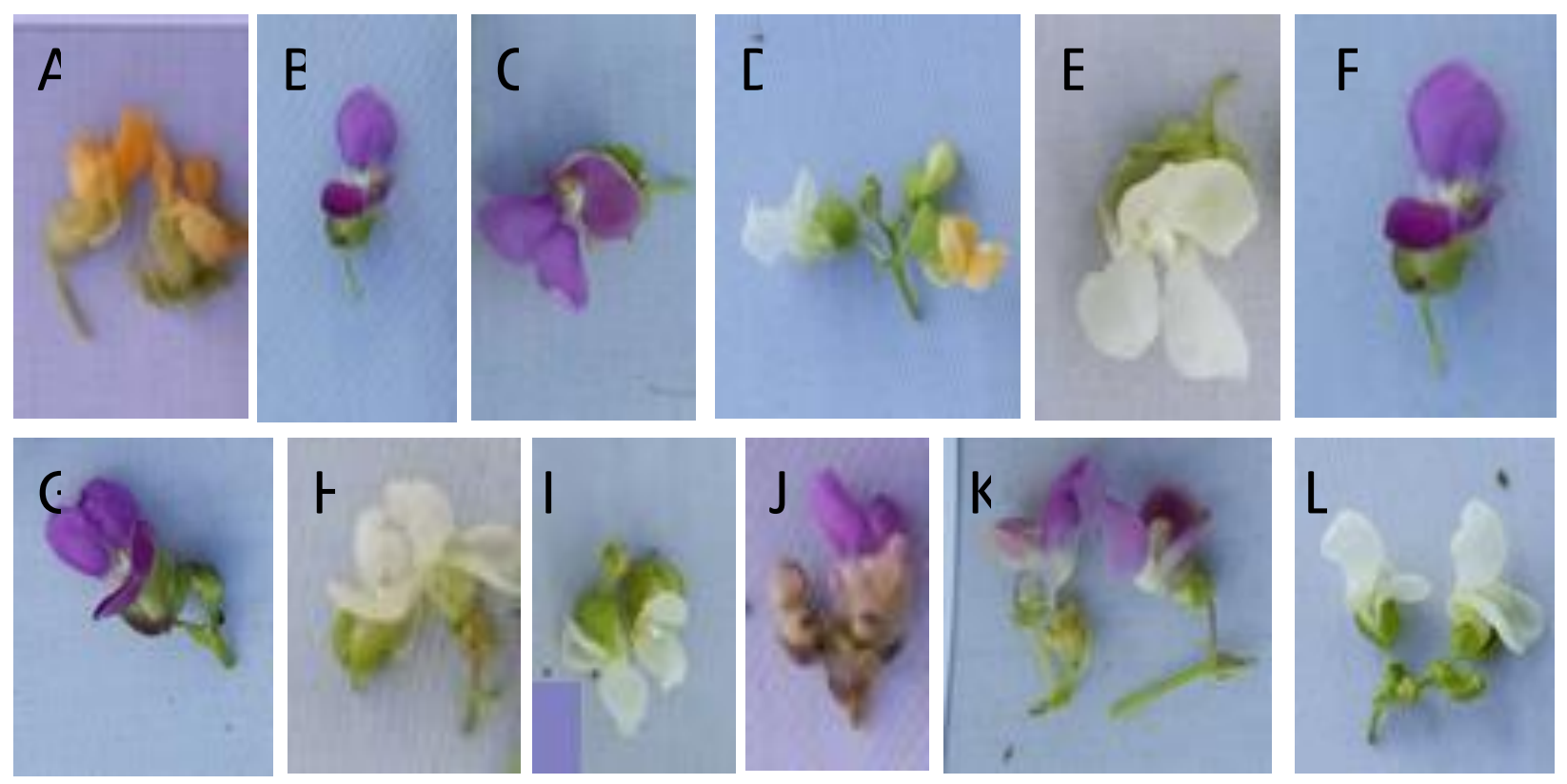

Figure3. Flower morphology of pole-type French bean genotypes evaluated at HRS, Dailekh, 2018/19. A: Bhatte; B: Chaumase; C: DhankuteChhirke; D: WP Con Bean; E: White OP; F: Dhundi Raj; G: LB-31; H: LB-37; I: LB-39; J: Madhav; K: Chinese Long; and L: Trishuli 
The seed color was also varied from light brown, black, and grey to white type (Figure3). Similarly, seed shape was varied from circular elliptic, elliptic, to kidney type in the genotypes.
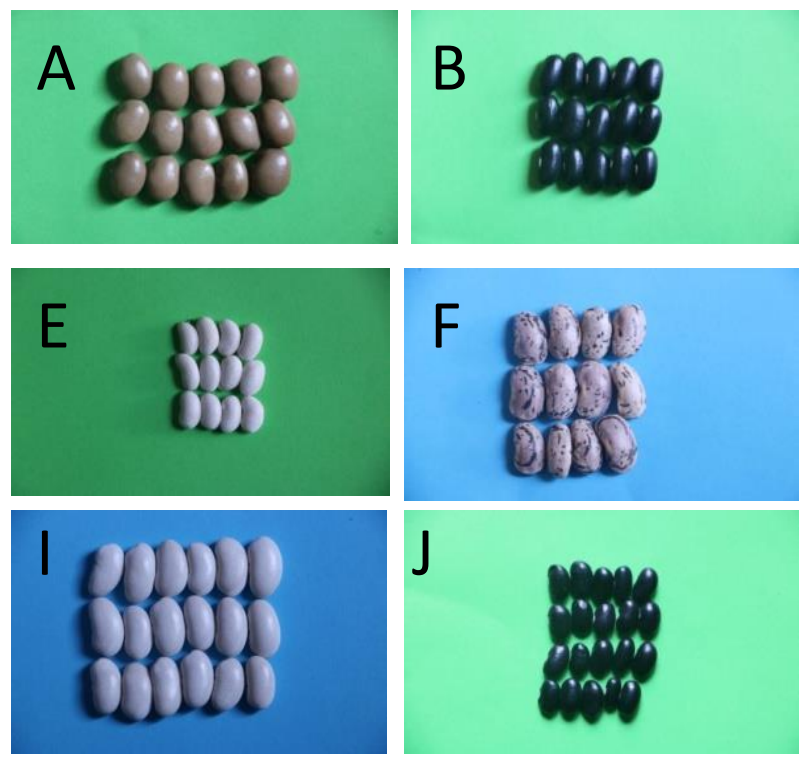
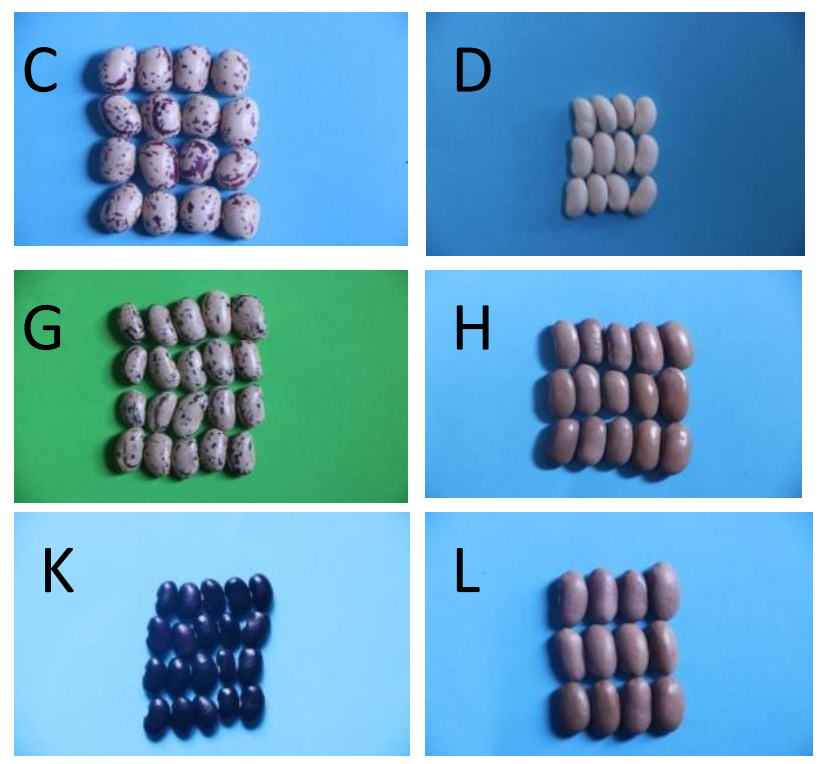

Figure3. Seed morphology of pole-type French bean genotypes evaluated at HRS, Dailekh, 2018/19. A: = Bhatte; B: Chaumase; C: DhankuteChhirke; D: WP Con Bean; E: White OP; F: Dhundi Raj; G: LB-31; H: LB-37; I : LB-39; J: Madhav; K:Chinese Long; and L: Trishuli

\section{DISCUSSION}

Pole-type French bean genotypes showed the significant differences in plant height, and number of pod per plant. The significant variation in plant height found in this study might be due to genetic make-up of cultivar and environmental differences and similar findings were reported by Noor et al (2014). Hussain (2005) also reported significant variation in plant height in bush bean genotypes. Plant height in beans was influenced by the genotype (Neupane et al 2008, Pandey et al 2011). The significant variation observed in cluster number per plant and pod number per plant in the years due to variation in temperature and rainfall (Figure1). The significant variation among the genotypes for green pod number per plant had been reported by many researchers (Akhilesh et al 2013, Muthuramu et al 2015, Yohannes et al 2020). The variation in number of green pod per plant might also be due to differences in inflorescences, pods number per racemeand flower dropping tendency of the genotypes (Khan 2003).

Significant variation was observed in green pod weight per pod, green pod weight per plant, green pod weight per plot and green pod yield ton per hectare. The green pod yield variation amongst the genotypes under varying field conditions had been reported by several workers (Hari and Singh 1990, Muthuramu et al 2015). Cluster number per plant, green pod number per plant, green pod weight per pod, green pod weight per plant is attributed to high green pod yield. Our study exhibited the fresh greed pod yield varied from $17.6 \mathrm{t} / \mathrm{h}$ a to $36.1 \mathrm{t} / \mathrm{ha}$ with the average of $25.6 \mathrm{t} / \mathrm{ha}$. In contrast, in the study of Angadi and Patil (2017), they reported that yield varied from $15.1 \mathrm{t} / \mathrm{ha}$ to 17.0 t/ha in pole type French bean genotypes. This might be due to genetic potential of variety.

Pod length, and diameter, dry pod number per plant, number of seed per pod and dry pod weight per plot, dry grain weight per plot, hundred grain weight and dry grain yield revealed significant variation among the genotypes. In our study, pod length was varied from $10.8 \mathrm{~cm}$ to $22.3 \mathrm{~cm}$ with the average of $16.6 \mathrm{~cm}$ but in the study of Neupane et al (2008), they reported the varying pod lengths ranging from 6.7 to $17.4 \mathrm{~cm}$ whereas in the study of Pandey et al (2011), they reported $11.5 \mathrm{~cm}$ to $20.4 \mathrm{~cm}$ in pole-type French bean 
genotypes. Islam et al (2010) reported that considerable variation in pod length varying from $3.96 \mathrm{~cm}$ to $18.20 \mathrm{~cm}$ in hyacinth bean. In this study, average pod diameter recorded was $10.5 \mathrm{~mm}$ but in the study of Pandey et al (2011), they reported $33.5 \mathrm{~mm}$ pod width in pole-type in Tarbarebean. Neupane et al (2008) reported that pod length and width in beans were influenced by the genotype. Genotypic variation was observed in dry seed number per pod, dry pod weight per plot, dry grain weight per plot, hundred grain weight and dry grain yield. The number of dry seed per pod was significantly different among the genotypes which were similar to the result of Noor et al (2014). In this study, grain yield was varied from 1.1 to 2.4 t/ha with an average of $1.8 \mathrm{t} / \mathrm{ha}$ which is close to the findings of Chowdhury and Faruque (1973).

Correlation analysis measures the mutual relationship among the agronomic traits and determines the components characters on which selection can be used for improvement in yield (Singh 1990). In this study, number of clusters per plant, pod number per plant, green pod weight per pod, and green pod weight per plot showed positive and significant correlation with green pod yield. Dursun (2007) reported positive correlation of pod number per plant with green pod yield in snap bean. In the study of Shah et al (1999), they reported significant and positive association between green pod weight and green pod yield. Green pod length showed significant association with green pod yield which were reported by previous researchers (Shaban 2005, Dursun 2007). Dry pod number per plant, seed number per plant, dry grain weight and hundred grain weight exhibited positive and significant association with dry grain yield. The positive and significant association of number of seeds per pod with seed yield had been reported by Kamal and Ahmed (2011). Genotypes with high pod weight and pod length, hundreds grain weight and numbers seeds per pod should be used to improve grain yield in cowpea (Carvalho et al 2012).

The present study showed the variation in flower color, pod beak orientation, pod color, pod quality, seed color and shape in French bean genotypes. In the study of Pandey et al (2011), they had also reported the variation in flower, pod and seed color and shape in pole-type French bean genotypes. Islam et al (2010) reported white, violet and pink flower in hyacinth bean genotypes. Our study showed that most of the genotypes produced green pod color which is important trait for marketing purpose and this is very close to the findings of Muchui et al (2008). In our study, seed color was varied from light brown, black, grey, mottled, brown to white and diversity of seed color in hyacinth bean and French bean were reported by Stoilova et al (2005) and Islam et al (2010), respectively. The presence of varied seed color and shape found in pole-type French bean genotypes indicates the wider diversity in phenotypic characters.

\section{CONCLUSION}

This study evaluated the plant morphological and yield traits and, association among the agronomic traits in diverse pole-type French bean genotypes at Karnali province of Nepal. Analysis of variance revealed that French bean genotypes showed significant variation for all the characters except few insignificant traits. Result of experiment showed that genotype LB-31 gave the highest greed pod yield $(36.1 \mathrm{t} / \mathrm{ha})$ and grain yield (2.4 t/ha). Furthermore, genotypes Chaumase and LB-39 produced the highest greed pod yield (31.3 $\mathrm{t} / \mathrm{ha}$ and $31.2 \mathrm{t} / \mathrm{ha}$, respectively). In addition, genotypes studied in the experiment were all well adapted to the study area and therefore, high yielding genotypes viz LB-31, Chaumase and LB-39 could be directly used as seed sources for production of French bean. Cluster number per plant, pod number per plant, green pod weight per pod, and green pod weight per plant showed significant positive association with green pod yield and dry grain yield so selection of these traits should be done to enhance the yield potential in French bean genotypes. In addition, dry pod number per plant, seed number per pod, dry grain weight and hundred grain weights can be considered the important traits for selection to improve the grain yield of French bean.

\section{ACKNOWLEDGEMENTS}

The first author thanks Nepal Agricultural Research Council (NARC) for financial support under variety development project of HRS, Dailekh. We thank all the field staffs of HRS, Dailekh for their support to conduct field experiment and to take observations during the research period. 


\section{REFERENCES}

Akhilesh S, GD Sharma, Y Singh, M Sharma, V Katoch and KC Sharma. 2013. Optimum sowing dates and varieties for seed productivity of pole French bean (Phaseolus vulgaris L.) under north western Himalayas. African J Agric. Res. 8 (48): 6196-6201.

Alemu Y, S Alamirew and L Dessalegn. 2017. Correlation and path analysis of green pod yield and its component in snap bean (Phaseolus vulgaris L.) genotypes. Int J Agric For 4:30-36.

Angadi P and MG Patil. 2017. Evaluation of Pole type French bean genotypes under Raichur Region. Int. J. Curr. Microbiol. App. Sci. 6 (11): 2645-2650.

Carvalho LCB, KJD eSilva, M deMoura Rocha, MB de Sousa, C de Jesus Pires and JA Rodrigues Nuns. 2012. Crop Breed ApplBiotechnol12: 211-214.

Chowdhury ATMS and AHM Faruque. 1973. Pinto, a new variety of bean for Bangladesh. Bangladesh Hort. 1: 37 38

Dursun A. 2007. Variability, heritability and correlation study in bean (Phaseolus vulgaris L.) genotypes. J. Agri. Sci. 3(1): 12-16.

Goutam P, S Mulani and G Arvind. 2001. Evaluation of genetic diversity in some pea varieties grown in NorthEastern hills. Indian Journal of Hill Farming. 6 (2): 27-28.

Hari H, and BP Singh. 1990. Evaluation of French bean (Phaseolus vulgaris L.) germplasm. Veg. Sci. 17 (1): 47-55.

Hussain MM. 2005. Yield and quality of bush bean (Phaseolus vulgaris L.) genotypes as influenced by date of sowing. Master of Science (M.S.) Thesis, Department of Agronomy, BangabandhuSeikhMujibur Rahman Agricultural University (BSMRAU), Salna, Gazipur.

IBPGR. 1982. Descriptor List for Phaseolus vulgaris L. International Board for Plant Genetic Resources, Rome.

Islam MS, MM Rahman and T Hossain. 2010. Physico-morphological variation in hyacinth bean (Lablab purpureus L.). Bangladesh J. Agri. Res. 35 (3): 431-438.

Kamal U and S Ahmed. 2011. Variability, correlation and path analysis for seed yield and yield related traits in common beans. Indian J. Hort. 68 (1): 56-60.

Khan MMR. 2003. Performance of lablab bean genotypes under different supports. MS Thesis, Department of Horticulture, BSMRAU, Salna, Gazipur.

MoAD. 2018. Statistical information on Nepalese Agriculture 2017/18 (2074/75). Ministry of Agriculture and Livestock Development, Singhadurbar, Kathmandu, Nepal.

Muchui MN, ANdegwa, S Wachiuri, B Muthama and J Kimamira. 2008. Postharvest evaluation of introduced French bean (Phaseolus vulgaris L.) varieties. Afr. J. Hort. Sci. 1: 116-120.

Muthuramu S, VK Paulpandi, S Sakthievel, K Ramakrishnan and R Karthik. 2015. Assessing the performance of French bean (Phaseolus vulgaris L.) in District Virudhunagar of Tamilnadu. J KrishiVigyan3(2): 5-7.

Neupane RK and ML Vaidya. 2002. Development of improved production technology of Phaseolus bean to the hills of mid-western Nepal. In: Proceedings of the First Stakeholders Meeting, NGLRP, Rampur, Nepal; pp. 5-11.

Neupane RK, R Shrestha, ML Vaidya, EM Bhattarai and R Darai. 2008. Agro-morphological diversity in common bean (Phaseolus vulgaris L.) landraces of Jumla, Nepal. In: Proceedings of the Fourth International Food Legumes Research Conference. New Delhi, India; pp. 639-648.

Noor F, F Hossain and U Ara. 2014. Screening of French bean (Phaseolus vulgaris L.) genotypes for high yield potential. Bangladesh J. Sci. Ind. Res. 49 (4): 227-232

Pandey YR, DM Gautam, RB Thapa, MD Sharma and KP Paudyal. 2011. Variability of French bean in the Western mid hills of Nepal. Kasetsart J. (Nat. Sci.) 45: 780-792.

Shaban N. 2005. Analysis of the correlation and regression coefficient of the interaction between yield and some parameters of snap beans plants. J. Sci. 3(6): 27-31

Shah D, CC Pant and SS Solanki. 1999. Studies on agronomic traits and genetic parameters in French bean (Phaseolus vulgaris L.). Progressive Horticulture. 31 (3-4): 194-198.

Singh BD. 1990. Plant breeding, principles and methods. Kalyani Publishers, New Delhi.

Singh, U and B Singh. 1992. Tropical grain legumes as important human foods. Economic Bot. 46 (3): 310-321.

Stoilova T, G Pereira, and MM Tavares de Sousa and V Carnide. 2005. Diversity in common bean landraces (Phaseolusvulgaris L.) from Bulgaria and Portugal. J. Central European Agric. 6 (4): 443-448.

Swaider JM, GW Ware and JP McCollum. 1992. Producing Vegetable Crops. $4^{\text {th }}$ Ed. Interstate Publishers USA. Yohannes, S, G Loha and MK Gessese. 2020. Performance evaluation of common bean (Phaseolus vulgaris L.) genotypes for yield and related traits at Areka, Southern Ethiopia. Adv Agric, 2020: 1-8.

$|1------||-------| \mid$ 